\title{
1 A stepwise route to domesticate rice by controlling seed 2 shattering and panicle shape
}

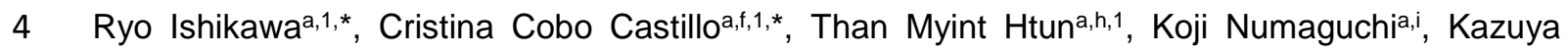

5 Inoue $^{b}$, Yumi Oka ${ }^{a}$, Miki Ogasawara ${ }^{a}$, Shohei Sugiyama ${ }^{a}$, Natsumi Takama ${ }^{a}$, Chhourn Ornn ${ }^{a, j}$,

6 Chizuru Inoue ${ }^{a}$, Ken-ichi Nonomurac,d, Robin Allabye, Dorian Q Fuller ${ }^{\mathrm{f}, \mathrm{g}}$ and Takashige Ishiia

7 aLaboratory of Plant Breeding, Graduate School of Agricultural Science, Kobe University; Kobe,

8 657-8501, Japan

9 bLaboratory of Hydraulic Structures and Geo-Environmental Engineering, Graduate School of

10 Agricultural Science, Kobe University; Kobe, 657-8501, Japan

11 cLaboratory of Plant Cytogenetics, National Institute of Genetics; Mishima, Shizuoka, 411-8540,

12 Japan

13 dDepartment of Genetics, School of Life Science, The Graduate University for Advanced Studies

14 (SOKENDAI); Mishima, Shizuoka, 411-8540, Japan

15 eSchool of Life Sciences, University of Warwick; Coventry, CV4 7AL, UK

16 Institute of Archaeology, University College London; London, WC1H OPY, UK

17 9School of Cultural Heritage, Northwest University, Shaanxi, 710069, China

18 hPresent address: Department of New Genetics, Advanced Center of Agricultural Research and

19 Education, Yezin Agricultural University; Yezin, Nay Pyi Taw, 15013, Myanmar

20 iPresent address: Japanese Apricot Laboratory, Wakayama Fruit Tree Experiment Station;

21 Wakayama, 645-0021, Japan

22 jPresent address: Plant Breeding Division, Cambodian Agricultural Research and Development

23 Institute (CARDI); Phnom Penh, Cambodia 
251 These authors contributed equally to this work.

26 * To whom correspondence may be addressed.

27 Email: Ryo Ishikawa (r-ishika@port.kobe-u.ac.jp) or Cristina Cobo Castillo (riscastillo@me.com)

29 Author Contributions:

30 R.I. and C.C.C. designed the research; R.I., C.C.C., T.M.H, Y.O., M.O., S.S., N.T., C.O., and C.I. 31 conducted genetic analysis; K.N. and R.A. carried out population genetic analysis; C.C.C. carried out seed-gathering experiment; C.I. conducted SEM analysis; K.I. and R.I. carried out structural

33 mechanic simulation; K-I.N. contributed wild rice materials and their evaluation; R.I., C.C.C., R.A., 34 D.F., and T.I. wrote the manuscript.

Competing Interest Statement:

37 The authors declare no conflict of interest.

39 Classification:

40 Biological Sciences/Plant Biology or Biological Sciences/Anthropology

42 Keywords:

43 Oryza sativa, Oryza rufipogon, domestication, seed shattering, closed panicle

This PDF file includes:

Main Text

Figures 1 to 5 . 


\section{Abstract}

63

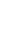

4

85 86

Rice (Oryza sativa L.) is consumed by more than half of the world's population, but despite its global importance the mechanisms of domestication remain unclear. During domestication, wild rice (O. rufipogon Griff.) was transformed by acquiring non-seed-shattering behaviour, an important genetic change that allowed humans to increase grain yield. However, we show previously identified loci, sh4 and $q S H 3$, are individually insufficient to explain loss of seed shattering nor increases in harvest yield in wild rice. We identify the complementary interaction of key mutations for abscission layer interruption and panicle architecture that were causal in the early domestication of Asian rice. An interruption of abscission layer formation requires both sh4 and qSH3, which presents an apparent barrier to selection of shattering loss. We identified the causal single nucleotide polymorphism at $q S H 3$ within a seed-shattering gene OsSh1 conserved in indica and japonica subspecies, but absent in the circum-aus group of rice. We demonstrate through harvest experiments that seed-shattering alone does not significantly impact yield. Instead, we observed yield increases under a SPR3-controlled closed panicle formation, which is augmented by the integration of sh4 and $q S H 3$ alleles causing a slight inhibition of abscission layer. Complementary manipulation of seed shattering and panicle shape result in a panicle structure that is mechanically stable. We propose a stepwise route in the earliest phase of rice domestication in which selection for visible SPR3-controlled closed panicle morphology was instrumental in the sequential recruitment of sh4 and $q S H 3$ and leading to loss of shattering.

(1)




\section{Significance Statement}

89

90

91 rice, previously attributed to single mutations such as in sh4, is considered the principal genetic

92 change which resulted in yield increases. However, we show that sh4 is insufficient on its own to

93 cause abscission layer disruption and other genes, such as $q S H 3$ are required, making

94 mechanisms for the initial selection of non-shattering unclear. We show that shattering loss in wild

95 rice genetic backgrounds does not increase yields. We identify an interaction in which a second

96 trait, closed panicle formation controlled by SPR3, both increases yield and facilitates recruitment

97 of sh4 and $q S H 3$ which synergistically augment yield, leading to a stepwise route for rice 98 domestication. 


\section{Main Text}

117

118

119

120

121

122

123

124

125

126

127

128

129

130

\section{Introduction}

Selection of naturally occurring variations in wild plants that provided useful agronomic traits was an essential step for crop domestication. These traits are often related to yield, such as seed number, seed size and a loss of seed dispersal, as well as ease of cultivation, including plant architecture, seed dormancy, and photoperiod sensitivity. Plants with these traits provided the necessary impetus for humans to shift from hunting and gathering to cultivating. The visible morphological changes were key for triggering domestication in many crop species. Among several domestication-related traits, the suppression of seed shattering is considered the most important genetic change that allowed humans to increase grain yield and differentiated domesticated from wild plants $(1,2)$.

Rice (Oryza sativa L.) is consumed by almost half of the world's population and is particularly important in Asia. During its domestication, wild rice (O. rufipogon Griff.) was transformed by acquiring non-seed-shattering behaviour. Investigations on prehistoric rice spikelet bases from archaeological sites demonstrate that suppression of seed shattering leaves a phenotypic trace that can be observed through the examination of the abscission layer (3). Archaeological rice spikelet bases with rough and deeply torn scars in the spikelet base (rachilla) indicate that humans had to actively detach the spikelets from the pedicels by threshing. More than a decade ago, two loci, $q S H 1$ and sh4, were identified as major loci that influence the reduction in seed shattering based on quantitative trait locus (QTL) analyses $(4,5)$. Since sh4 and $q S H 1$ displayed large phenotypic variances, they were recognised as crucial to rice domestication.

To better understand how $q S H 1$ and sh4 were involved in the loss of seed shattering in wild rice, we developed introgression lines (ILs) named IL( (SSH1-N) and IL(sh4-N), which contain small chromosomal segments from $\mathrm{O}$. sativa Nipponbare at the $q \mathrm{SH} 1$ and sh4 loci, respectively, in the genetic background of wild rice O. rufipogon W630 (6). Complete seed shattering was still observed in these lines, indicating that neither of the single mutations at $q S H 1$ and sh4 could account for non-shattering during rice domestication. As the sh4 mutation is commonly observed 
145 in cultivated rice, and the qSH1 mutation is only found in some japonica cultivars (4), additional

146 mutation(s) together with sh4 must have played a role in reducing seed shattering during the early

147 stages of rice domestication.

148 In this study, we conducted genetic analysis of the qSH3, an additional locus involved in

149 the loss of seed shattering together with sh4, and identified the causal mutation selected during

150 rice domestication. The frequency of the $q S H 3$ alleles in rice cultivars and their effects on the loss

151 of seed shattering were evaluated. In addition, we assessed the interaction between seed

152 shattering and panicle shape, and also investigated their role in seed shattering behaviour based

153 on structural mechanic analysis to better understand the selection process on a loss of seed

154 shattering in rice.

Identification of the causal mutation at $q S H 3$, a locus involved in the loss of seed shattering during rice domestication

To identify additional genomic regions involved in reducing seed shattering, we produced

162 background of wild rice, O. rufipogon W630 (7). IL(qSH1-N+sh4-N) was crossed with Nipponbare,

163 and their $\mathrm{F}_{2}$ population was subjected to QTL analysis to determine the degree of seed shattering,

164 and the locus $q S H 3$ was identified $(7,8)$. The effect of $q S H 3$ was confirmed in the wild genetic

165 background as IL $(q S H 1-\mathrm{N}+s h 4-\mathrm{N}+q S H 3-\mathrm{N})$ showed reduced seed-shattering behaviour compared

166 with IL(qSH1-N+sh4-N) (7). To identify the gene for $q S H 3$, we performed high-resolution linkage

167 analysis using a mapping population from a cross between IL( qSH1-N+sh4-N) and Nipponbare (9)

168 (Fig. S1). The genomic location of $q S H 3$ was delimited by the two recombinants to a 1,228 bp

169 region that harboured a part of a previously known seed-shattering gene, OsSh1 (Os03g0650000)

170 (Figs. S2 and S3). This gene was identified as a homolog of Sh1, which controls abscission layer

171 formation in sorghum (10). The function of OsSh1 in seed shattering was studied using artificially

172 induced rice materials showing that the null mutations caused a complete loss of seed shattering 
$173(10,11)$, but the causal mutation selected in rice domestication was not determined. A sequence

174 survey in the 1,228 bp region of $5^{\prime}$ UTR, exon 1 , and intron 1 of OsSh1 identified seven 175 polymorphisms between Nipponbare and W630 (Fig. 1A and Table S1). Among them, only a single 176 nucleotide polymorphism (SNP), namely SNP-70, was located in the coding region (exon1) of 177 OsSh1, causing an amino acid substitution from leucine in W630 to phenylalanine in Nipponbare 178 (Fig. S4). To test whether SNP-70 is associated with the degree of seed shattering, we performed 179 a transformation test in the Nipponbare genetic background of two constructs that differed only at 180 the SNP-70 position. In the preliminary experiment, we observed that $\mathrm{IL}(q S H 3-\mathrm{W})$, carrying the 181 W630 allele at qSH3 in Nipponbare genetic background (Fig. S5), showed a non-seed-shattering 182 behaviour similar to Nipponbare, whereas IL $(q S H 1-W+q S H 3-W)$ displayed a significantly higher 183 seed-shattering degree than the IL( SSH1-W) (Fig. 1B and Fig. S6). These findings indicate that the 184 seed-shattering effect of $q S H 3$ was considerably enhanced by a functional allele at $q S H 1$. 185 Therefore, we introduced two types of constructs carrying the qSH3 cDNA sequence of W630 $186\left(q S H 3^{w 630}\right)$ and Nipponbare $\left(q S H 3^{N p b}\right)$ into IL( $\left.q S H 1-W\right)($ Fig. 1C). As a result, transformants with $187 q \mathrm{qSH} 3^{W 630}$ showed enhanced seed shattering, compared with the control IL( $\left.q S H 1-\mathrm{W}\right)$, whereas 188 those with $q S H 3^{N p b}$ did not (Fig. 1D), confirming that SNP-70 is the causal mutation for reduced 189 seed shattering.

Causal mutation at $q S H 3$ is conserved in both japonica and indica but not in circum-aus rice cultivars

Next, we analysed the distribution of SNP-70 at $q S H 3$ in cultivated rice. First, genotyping at the causal SNPs of $s h 4, q S H 1$, and $q S H 3$ was conducted for the three rice cultivars, Nipponbare, 195 IR36, and Kasalath, belonging to japonica, indica, and circum-aus, respectively. All cultivars 196 possessed the causal mutation at sh4 and only Nipponbare had the causal mutation at qSH1. As 197 for $q S H 3$, Nipponbare and IR36 carried the causal mutation, but it was absent in Kasalath (Fig. 2A). 198 We further found that the Kasalath haplotype is similar to that of W630 around the qSH3 region 199 (Table S1). Using the diverse varieties of the World Rice Core Collection (WRC) (12), we found 
200 that 14 lines, all belonging to circum-aus, carried the functional allele at qSH3 (Table S2 and Figs.

201 S7 and S8). Furthermore, the Rice 3K genome project data (13) clearly showed that both indica

202 and japonica carried the causal mutation, but almost $90 \%$ of circum-aus rice carried a functional 203 allele at qSH3 (Fig. 2B). To further understand the footprint of qSH3 selection, we analysed 204 nucleotide diversity across the qSH3 genomic region. Using sequences of the Rice $3 \mathrm{~K}$ genome 205 collection (13), we detected a selective sweep at $q S H 3$ in both indica and japonica, but not in the 206 circum-aus linage (Fig. 2C, Figs. S9 and S10). These results suggest that circum-aus may have 207 experienced a separate trajectory to evolve reduced seed shattering (14). Thus, the reduction in 208 seed shattering is dependent on lineage-specific variations in the subspecies, which is key to 209 understanding the processes of rice domestication.

\section{Role of $q S H 3$ causal mutation in an initial loss of seed shattering}

We next aimed to understand how the causal SNP at $q S H 3$ contributed to initial rice 213 domestication by reducing seed shattering in japonica and indica. Since the sh4 mutation is 214 conserved in all cultivated rice, we produced IL(sh4-N) and IL ( $q S H 3-\mathrm{N})$ in the genetic background 215 of wild rice O. rufipogon W630. Complete formation of the abscission layer similar to W630 was 216 observed in both ILs (Fig. 3), suggesting that the single mutation at each locus was insufficient for 217 phenotypic change in the abscission layer formation. However, a slight inhibition of the abscission

218 layer around vascular bundles was observed in IL(sh4-N+qSH3-N) (Fig. 3). A slight abscission 219 layer inhibition was also observed in several wild rice accessions of $O$. rufipogon carrying mutations 220 at both sh4 and $q S H 3$ (Figs. S11 and S12, and Table S3), although they may have gained these 221 domestic-type alleles through introgressions from cultivated rice (15). Even if the mutations in the 222 two loci displayed phenotypic difference in the abscission layer formation adequate to reduce 223 shattering under a greenhouse conditions without wind, but their effect on seed shattering was 224 much less than expected under field conditions. 
226 Seed-shattering behaviour associated with a slight inhibition of abscission layer was

227 mitigated by a closed panicle formation

228 Previously, we reported that a closed panicle trait controlled by OsLG1 had a major effect

229 in rice domestication by facilitating grain harvest (1). A closed panicle reduces seed shedding by

230 retaining seeds that get stuck by long awns found in the lower sections of the panicle. Interestingly,

231 the SPR3 (a locus regulating OsLG1) region was under strong selection in indica, japonica, and

232 circum-aus (Fig. S13), suggesting that the closed panicle trait was selected in the early phase of

233 rice domestication and so might have been associated with seed-shattering changes, although this

234 trait, unlike seed shattering, is not visible archaeologically. Therefore, we generated seven wild ILs

235 with different combinations of the three loci (sh4, qSH3, and SPR3) (Fig. S14) and compared their

236 morphology together with the wild rice O. rufipogon W630 (Fig. S15). The heading date of all ILs

237 was similar to those of W630 (Fig. S16), but with distinct abscission layer formation (Fig. 3 and Fig.

238 S17) and panicle structure (Figs. 4A and 4B). Small BTS values were observed only for IL(sh4-

$239 \mathrm{~N}+q S H 3-\mathrm{N})$ and IL(sh4-N+qSH3-N+SPR3-N), while the rest were close to zero as wild rice (Fig.

$2404 \mathrm{C})$. We were interested in assessing the differences in yields brought about by the causal

241 mutations and which combination of alleles would have provided humans in prehistory with higher

242 yields, including whether a closed panicle conferred additional value to humans when gathering

243 wild rice. We therefore subjected the seven ILs and W630 to a seed-gathering experiment in the

244 field (Fig. S18, Movies S1 and S2). The seed-gathering rate from IL(sh4-N) and IL(qSH3-N), and

$245 \mathrm{IL}($ sh4-N+qSH3-N) was not significantly different from that of W630 (Fig. 4D and Table S4).

246 However, the three ILs with a closed panicle structure, IL(SPR3-N), IL(sh4-N+SPR3-N), and

247 IL(qSH3-N+SPR3-N), presented a slightly increased yield. In contrast, IL(sh4-N+qSH3-N+SPR3-

$248 \mathrm{~N}$ ), with a combination of closed panicle and abscission layer inhibition, showed a significant

249 increase in gathering rate compared with W630 (Fig. 4D and Table S4). As closed panicles would

250 be easily visible in the field, humans could have targeted this higher yielding rice (Fig. S19).

251 When plants were sown from harvests, closed panicles would have been selected for, and

252 unintentionally would have selected also for reduced shattering, because of the enhanced 
253 gathering rate that would occur in the presence of the domesticated alleles. As these alleles

254 increased in frequency and became fixed this would have increased yields, which would have 255 encouraged further investment in rice cultivation (16). The selection for closed panicle instigates 256 self-pollination behaviour owing to the long awns, which disturb the free exposure of anthers and 257 stigmas (stigmas and closed panicle) (17). Thus, a closed panicle may also be advantageous in 258 mitigating natural variations in seed-shattering loci by reducing outcrossing. Although awns in 259 modern cultivated rice are undesirable, gathering of rice in the early stages of rice domestication 260 might have benefitted from the presence of awns in plants with closed panicles, as these would 261 have increased yield and self-pollination rates.

\section{Complementary interaction of a slight inhibition of abscission layer and closed panicle} formation synergistically contributed to structural stability of panicle to increase yield

To better understand how the closed panicle, caused by SPR3, and the inhibition of abscission layer, caused by sh4 and $q S H 3$, contributed to the initial loss of seed shattering, we analysed their roles by performing structural mechanics analysis. The awns of wild rice play a pivotal role in seed dispersal in spreading panicles (Fig. 5A). We measured the lengths and weights of awns and grains in wild rice O. rufipogon W630, as well as calculated the sectional force exerted on the spikelet base depending on panicle angles (Fig. 5B). The axial and shear forces were slightly

271 increased in a closed panicle compared to an open panicle (Fig. S20). However, the bending 272 moment, which is the predominant factor affecting seed dispersal in an open panicle, was 273 considerably reduced in a closed panicle (Fig. S20). A slight inhibition of the abscission layer by 274 sh4 and qSH3 led to an increase in the length of abscission layer inhibition (Fig. 3). Therefore, we 275 measured the length of the abscission layer and central vascular bundle in O. rufipogon W630 by 276 scanning microscopy (Figs. 5C and D). We also calculated the moment of inertia of the area, a 277 property that describes the torque required to break the abscission layer and so disarticulate the 278 grain, for the disrupted abscission layer (Fig. S20). The value increased exponentially with 279 increasing lengths of abscission layer inhibition. A reduction in the bending moment and an 
280 increase in the moment of inertia of the area act synergistically to reduce bending stress (Fig. 4E),

281 contributing to the structural stability of spikelets without shattering. Thus, the interaction between

282 a closed panicle and abscission layer inhibition acted complementarily to increase yield.

\section{Conclusions}

In this study, we identified key functional genes that contributed to rice domestication by increasing harvest yields. We identified the causal SNP at $q S H 3$ involved in reduced seed

287 shattering, whereas the previously proposed sh4 mutation alone could not trigger the non288 shattering morphology in rice $(6,17)$. We also explained how the early selection of a closed panicle 289 and the resulting mechanics of spikelet retention would have increased yields and facilitated the 290 selection for non-shattering. Our results showed that the initial step of rice domestication is more 291 complex than previously thought. With new information from our studies, a change in the long-held 292 perspective from a single domestication allele model to one where synergistic effects of several 293 domestication genes is needed.

294 The origin and spread of rice subspecies based on population genetic analysis have been 295 the subject of discussions $(14,18-21)$. However, most of the studies have been conducted using 296 the genome information of modern cultivars and wild rice, without considering the importance of 297 visible phenotypic changes targeted by humans. Therefore, the lineage-specific variations associated with quantitative traits are a key to further understand the process of rice domestication.

Based on our work, we propose a stepwise route for rice domestication. In wild rice, any of 300 the natural variations in the loci for seed shattering and closed panicle formation alone had little 301 effect on increasing yield (Fig. 4). Their combination, however, established an archaic rice which 302 could have been recognised by ancient gatherers as advantageous to increasing yields. The 303 change in harvesting efficiency along with the use of harvesting tools further promoted the 304 selections of natural variants in domestication-related traits in rice, a crop which now supports 305 billions of people worldwide. 
308

309

310

311

312

313

\section{Figure legends}

315

316

317

\section{Materials and Methods}

Details regarding plant materials, QTL mapping, fine mapping, transformation, population genetic analysis, histological analysis, seed gathering experiment, and structural mechanics analysis are provided in the Supplementary Information.

Fig. 1. Identification of a causal SNP of $q S H 3$ associated with the degree of seed shattering. (A) Fine mapping of the causal mutation at $q S H 3$ in a 1,228-bp region on chromosome 3. A causal SNP (SNP-70) identified in OsSh1 (Os03g0650000) is shown in bold. (B) Comparison of seedshattering degree by breaking tensile strength (BTS) values in three introgression lines, IL( $q S H 3-$ W), IL(qSH1-W), and IL $(q S H 1-\mathrm{W}+q S H 3-\mathrm{W})$ in the Nipponbare genetic background. Data are mean \pm s.d. of four plants. n.s. and ** indicate not significant and significant at the $1 \%$ level based on unpaired Student's t-test, respectively. (C) Two types of constructs carrying $q S H 3 \mathrm{cDNA}$ sequences of W630 and Nipponbare ( $q S H 3^{W 630}$ and $\left.q S H 3^{N p b}\right)$ driven by a 3-kb region of the promoter used for transgenic analysis. $(D)$ The BTS values observed for the transformants with $q S H 3^{W 630}$ and $q S H 3^{N p b}$. Black triangles represent the average BTS value of IL( $\left.q S H 1-\mathrm{W}\right)$.

\section{Fig. 2. Lineage-specific selection at $q S H 3$ locus in rice.}

(A) Genotyping at qSH3, sh4, and qSH1 for O. rufipogon W630, O. sativa japonica Nipponbare, indica IR36, and circum-aus Kasalath based on the causal SNPs by dCAPS markers. (B) Allele frequency of $q S H 3$ causal SNP (\%) in cultivated rice based on the 3K rice project data. Nipponbare type ('T') and W630 type ('C') are shown in yellow and green, respectively. (C) Nucleotide diversity ( $\pi$ ) observed for the domesticated rice in the physical position of 25.0-25.3 Mb on chromosome 3. A flanking region of the $q S H 3$ locus (around $25.2 \mathrm{Mb}$ ) was substantially decreased in japonica and indica cultivars. $\pi$ was calculated in 10-kb windows using SNPs data of the $3 \mathrm{~K}$ rice project (13).

\section{Fig. 3. Abscission layer formation partially inhibited by sh4 and $q S H 3$ in wild rice.}


336 Abscission layer formation in O. rufipogon W630, IL(sh4-N), IL(qSH3-N), IL(sh4-N+qSH3-N), and

337 O. sativa Nipponbare. Each enlarged section indicated by the dotted square is shown in the lower

338 panel. A black triangle indicates an inhibited area of the abscission layer observed for IL(sh4-

$339 \mathrm{~N}+q S H 3-\mathrm{N})$. Scale bars $=100 \mu \mathrm{m}$.

341 Fig. 4. Role of the sh4, $q S H 3$, and SPR3 mutations on the initial loss of seed shattering in 342 rice domestication.

$343(A, B)$ Panicle shape of the seven ILs at flowering $(A)$ and seed-maturation $(B)$ stages. Scale bars

$344=5 \mathrm{~cm}(C)$ Breaking tensile strength (BTS) values for the seven ILs. BTS values for O. sativa 345 japonica Nipponbare and indica IR36 are shown as control. n.s. indicates not significant by 346 unpaired Student's t-test. $(D)$ Seed-gathering rates (mean \pm s.d. of four plot replicates, with nine 347 plants in each plot) for O. rufipogon W630 and the seven ILs. Mean values labelled with different 348 letters are significantly different, whereas those with the same letters are not (Tukey's test with 349 arcsine transformed values, $P<0.05)$.

351 Fig. 5. Structural mechanics analysis of panicle shape and abscission layer inhibition for 352 the initial loss of seed shattering.

$353(A)$, Panicle shape of $O$. rufipogon W630. Bar $=5 \mathrm{~cm} .(B)$ Schematic representation of awn and 354 grain with panicle angle. $\theta$ represents the panicle angle. $l_{1}, l_{2}, m_{1} g$, and $m_{2} g$ indicate the length of 355 grain, awn, weight of grain and awn, respectively. (C) Detachment of grain and pedicel (left) and 356 scanning electron microscopy (SEM) analysis of the abscission layer of pedicel in O. rufipogon 357 W630 (right). AL and VB indicate abscission layer and vascular bundle, respectively. Bars $=1 \mathrm{~mm}$ 358 (left) and $100 \mu \mathrm{m}$ (right). (D) Schematic representation of the abscission layer. $D$ and $d$ indicate the 359 diameter of abscission layer and vascular bundles, respectively. Dotted circle indicates the area of 360 disrupted abscission layer. ( $E$ ) Simulation of the bending stress exerted on the spikelet base 361 depending on panicle shape and abscission layer inhibition, with higher stress experience in open 362 panicle with less inhibited abscission. 


\section{Acknowledgments}

The wild rice accessions of $O$. rufipogon were provided by the National Institute of Genetics supported by the National Bioresource Project, MEXT, Japan. We thank R. Morita and H. Fukayama, Kobe University, for their support on producing transgenic plants, and H. Furuumi, National Institute of Genetics, for growing wild rice accessions. This work was partly supported by Grants-in-Aid from the Japan Society for the Promotion of Science, 15KK0280 and 18K05594 (R.I.), JSPS overseas research program (C.C.C.), JSPS Bilateral Open Partnership Joint Research Project, No. JPJSBP120189948 (R.I., D.F.), Nikki Saneyoshi and Kinoshita foundations (R.I.), NIGJoint research (K-I.N, T.I.).

\section{References}

1. J. R. Harlan, J. M. de Wet, E. G. Price. Comparative evolution of cereals. Evolution 27, 311-325 (1973). doi.org/10.1111/j.1558-5646.1973.tb00676.x

2. J. F. Doebley, B. S. Gaut, B. D. Smith, The molecular genetics of crop domestication. Cell 127, 1309-1321 (2006). doi.org/10.1016/j.cell.2006.12.006

3. D. Q. Fuller, L. Qin Y. Zheng, Z. Zhao, X. Chen, L. A. Hosoya, G-P. Sun. The Domestication process and domestication rate in rice: spikelet bases from the lower Yangtze. Science 323, 1607-1610 (2009). doi.org/10.1126/science.1166605

4. S. Konishi, T. Izawa, S. Y. Lin, K. Ebana, Y. Fukuta, T. Sasaki, M. Yano. An SNP caused loss of seed shattering during rice domestication. Science 312, 1392-1396 (2006). doi.org/10.1126/science.1126410

5. C. Li, A. Zhou, T. Sang. Rice domestication by reducing shattering. Science 311, 19361939 (2006). doi.org/10.1126/science.1123604

6. R. Ishikawa, P. T. Thanh, N. Nimura, T. M. Htun, M. Yamasaki, T. Ishii. Allelic interaction at seed-shattering loci in the genetic backgrounds of wild and cultivated rice species. Genes Genet Syst 85, 265-271 (2010). doi.org/10.1266/ggs.85.265

7. T. M. Htun, C. Inoue, O. Chhourn, T. Ishii, R. Ishikawa. Effect of quantitative trait loci for seed shattering on abscission layer formation in Asian wild rice Oryza rufipogon. Breed Sci 64, 199-205 (2014). doi.org/10.1270/jsbbs.64.199

8. K. Onishi, K. Takagi, M. Kontani, T. Tanaka, Y. Sano. Different patterns of genealogical relationships found in the two major QTLs causing reduction of seed shattering during rice domestication. Genome 50, 757-766 (2007). doi.org/10.1139/G07-051

9. C. Inoue, T. M. Htun, K. Inoue, K. Ikeda, T. Ishii, R. Ishikawa. Inhibition of abscission layer formation by an interaction of two seed-shattering loci, sh4 and qSH3, in rice. Genes Genet Syst 90, 1-9 (2015). doi.org/10.1266/ggs.90.1

10. Z. Lin, X. Li, L. M. Shannon, C-T. Yeh, M. L. Wang, G. Bai, Z. Peng, J. Li, H. N. Trick, T. E. Clemente, J. Doebley, P. S. Schnable, M. R. Tuinstra, T. T. Tesso, F. White, J. Yu. Parallel domestication of the Shattering1 genes in cereals. Nat Genet 44, 720-724 (2012). doi.org/10.1038/ng.2281 
11. F. Li, A. Komatsu, M. Ohtake, H. Eun, A. Shimizu, H. Kato. Direct identification of a mutation in OsSh1 causing non-shattering in a rice (Oryza sativa L.) mutant cultivar using whole-genome resequencing. Sci Rep 10, 14936 (2020). doi.org/10.1038/s41598-02071972-1

12. N. Tanaka, M. Shenton, Y. Kawahara, M. Kumagai, H. Sakai, H. Kanamori, J. Yonemaru, S. Fukuoka, K. Sugimoto, M. Ishimoto, J. Wu, K. Ebana. Whole-genome sequencing of the NARO World Rice Core Collection (WRC) as the basis for diversity and association studies. Plant Cell Physiol 61, 922-932 (2020). doi.org/10.1093/pcp/pcaa019

13. W. Wang, R. Mauleon, Z. Hu, D. Chebotarov, S. Tai, Z. Wu, M. Li, T. Zheng, R. R. Fuentes, F. Zhang, L. Mansueto, D. Copetti, M. Sanciangco, K. C. Palis, J. Xu, C. Sun, B. Fu, H. Zhang, Y.Gao, X. Zhao, F. Shen, X. Cui, H. Yu, Z. Li, M. Chen, J. Detras, Y. Zhou, X. Zhang, Y. Zhao, D. Kudrna, C. Wang, R. Li, B. Jia, J. Lu, X. He, Z. Dong, J. Xu, Y. Li, M. Wang, J. Shi, J. Li, D. Zhang, S. Lee, W. Hu, A. Poliakov, I. Dubchak, V. J. Ulat, F. N. Borja, J. R. Mendoza, J. Ali, J. Li, Q. Gao, Y. Niu, Z. Yue, M. E. B. Naredo, J. Talag, X. Wang, J. Li, X. Fang, Y. Yin, J-C. Glaszmann, J. Zhang, J. Li, R. S. Hamilton, R. A. Wing, J. Ruan, G. Zhang, C. Wei, N. Alexandrov, K. L. McNally, Z. Li, H. Leung. Genomic variation in 3,010 diverse accessions of Asian cultivated rice. Nature 557, 43-49 (2018). doi.org/10.1038/s41586-018-0063-9

14. P. Civáň, H. Craig, C. J. Cox, T. A. Brown. Three geographically separate domestications of Asian rice. Nat Plants 1, 15164 (2015). doi.org/10.1038/nplants.2015.164

15. X. Jin, Y. Chen, P. Liu, C. Li, X. Cai, J. Rong, B-R. Lu. Introgression from cultivated rice alters genetic structures of wild relative populations: implications for in situ conservation. AoB Plants 10, plx055 (2017). doi.org/10.1093/aobpla/plx055

16. D. Q. Fuller. Transitions in productivity: rice intensification from domestication to urbanisation. Archaeol Int 23, 88-103 (2020). doi.org/10.14324/111.444.ai.2020.08

17. D. Q. Fuller. Transitions in productivity: rice intensification from domestication to urbanisation. Archaeol Int 23, 88-103 (2020). doi.org/10.14324/111.444.ai.2020.08

18. X. Huang, N. Kurata, X. Wei, Z-X. Wang, A. Wang, Q. Zhao, Y. Zhao, K. Liu, H. Lu, W. Li, Y. Guo, Y. Lu, C. Zhou, D. Fan, Q. Weng, C. Zhu, T. Huang, L. Zhang, Y. Wang, L. Feng, H. Furuumi, T. Kubo, T. Miyabayashi, X. Yuan, Q. Xu, G. Dong, Q. Zhan, C. Li, A. Fujiyama, A. Toyoda, T. Lu, Q. Feng, Q. Qian, J. Li, B. Han. A map of rice genome variation reveals the origin of cultivated rice. Nature 490, 497-501 (2012). doi.org/10.1038/nature11532

19. J. Y. Choi, A. E. Platts, D. Q. Fuller, Y-I. Hsing, R. A. Wing, M. D. Purugganan. The rice paradox: multiple origins but single domestication in Asian rice. Mol Biol Evol 34, 969-979 (2017). doi.org/10.1093/molbev/msx049

20. R. M. Gutaker, S. C. Groen, E. S. Bellis, J. Y. Choi, I. S. Pires, R. K. Bocinsky, E. R. Slayton, O. Wilkins, C. C. Castillo, S. Negrão, M. M. Oliveira, D. Q. Fuller, J. A. d'A. Guedes, J. R. Lasky, M. D. Purugganan. Genomic history and ecology of the geographic spread of rice. Nat Plants 6, 492-502 (2020). doi.org/10.1038/s41477-020-0659-6

21. X. Wei, J. Qiu, K. Yong, J. Fan, Q. Zhang, H. Hua, J. Liu, Q. Wang, K. M. Olsen, B. Han, $X$. Huang. A quantitative genomics map of rice provides genetic insights and guides breeding. Nat Genet 53, 243-253 (2021). doi.org/10.1038/s41588-020-00769-9

22. P. T. Thanh, P. D. T. Phan, N. Mori, R. Ishikawa, T. Ishii. Development of backcross recombinant inbred lines between Oryza sativa Nipponbare and O. rufipogon and QTL detection on drought tolerance and submergence avoidance Breed Sci 61, 76-79 (2011). doi.org/10.1270/jsbbs.61.76 
23. M. Kumagai, J. Kim, R. Itoh, T. Itoh. TASUKE: a web-based visualization program for largescale resequencing data. Bioinformatics 29, 1806-1808. doi.org/10.1093/bioinformatics/btt295

24. L. Mansueto, R. R. Fuentes, F. N. Borja, J. Detras, J. M. Abriol-Santos, D. Chebotarov, M. Sanciangco, K. Palis, D. Copetti, A. Poliakov, I. Dubchak, V. Solovyev, R. A. Wing, R. S. Hamilton, R. Mauleon, K. L. McNally, N. Alexandrov. Rice SNP-seek database update: new SNPs, indels, and queries. Nucl Acids Res 45, D1075-D1081 (2017). doi.org/10.1093/nar/gkw1135

25. S. Purcell, B. N., K. Todd-Brown, L. Thomas, M. A. R Ferreira, D. Bender, J. Maller, P. Sklar, P. I. W. de Bakker, M. J. Daly, P. C. Sham. PLINK: a tool set for whole-genome association and population-based linkage analyses. Am J Hum Genet 81, 559-575 (2007). doi.org/10.1086/519795

26. B. L. Browning, Y. Zhou, S. R. Browning. A one-penny imputed genome from nextgeneration reference panels. Am J Hum Genet 103, 338-348 (2018). doi.org/10.1016/j.ajhg.2018.07.015

27. Z. A. Szpiech, R. D. Hernandez. selscan: an efficient multithreaded program to perform EHH-based scans for positive selection. Mol Biol Evol 31, 2824-2827 (2014). doi.org/10.1093/molbev/msu211

28. A. Ferrer-Admetlla, M. Liang, T. Korneliussen, R. Nielsen. On detecting incomplete soft or hard selective sweeps using haplotype structure. Mol Biol Evol 31, 1275-1291 (2014). doi.org/10.1093/molbev/msu077

29. P. C. Sabeti, P. Varilly, B. Fry, J. Lohmueller, E. Hostetter, C. Cotsapas, X. Xie, E. H. Byrne, S. A. McCarroll, R. Gaudet, S. F. Schaffner, E. S. Lander, International HapMap Consortium. Genome-wide detection and characterization of positive selection in human populations. Nature 449, 913-918 (2007). doi.org/10.1038/nature06250 


\section{Fig. 1}

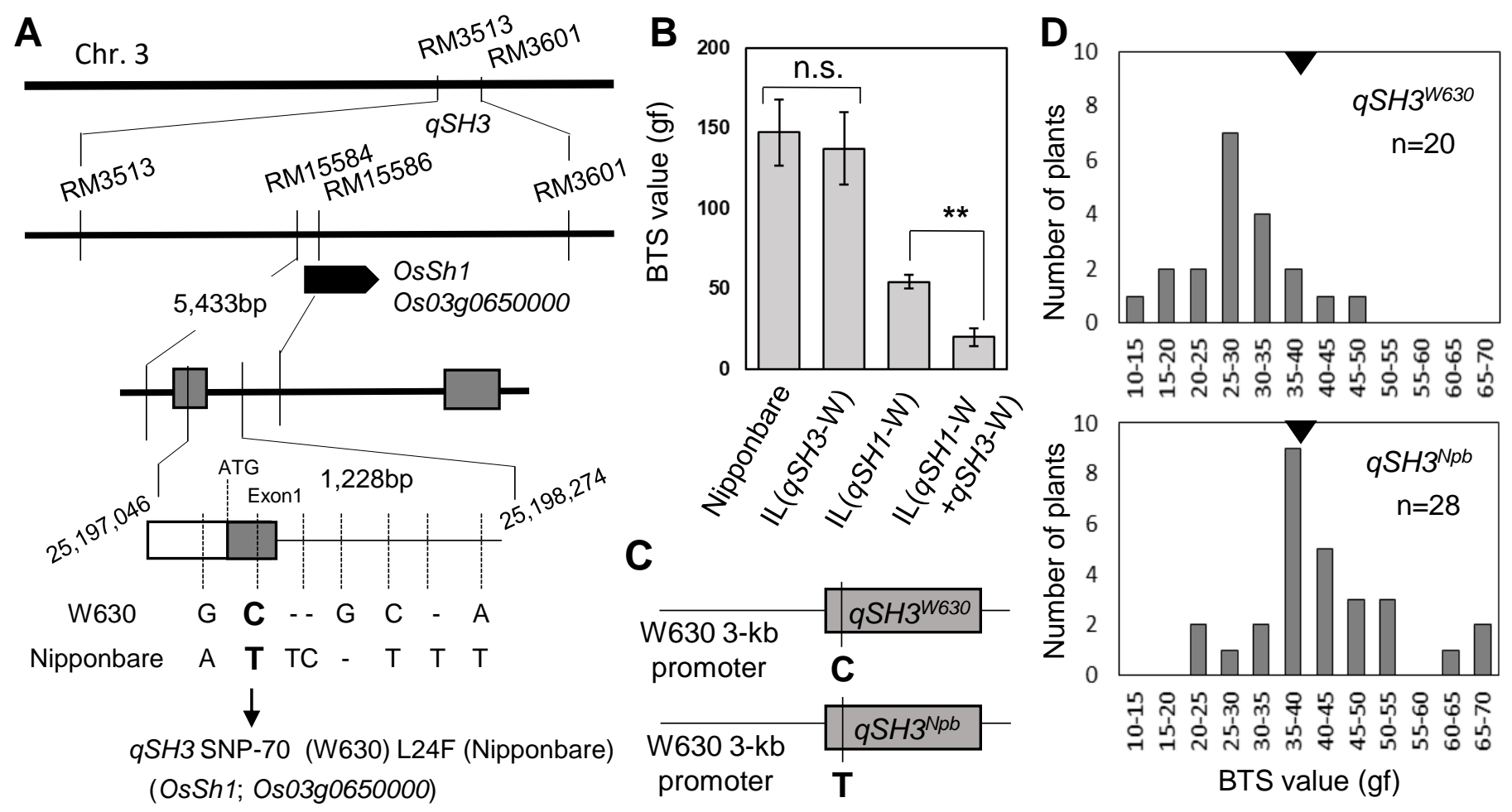

Fig. 1. Identification of a causal SNP of $q S H 3$ associated with the degree of seed shattering.

(A) Fine mapping of the causal mutation at $q S H 3$ in a 1,228-bp region on chromosome 3. A causal SNP (SNP-70) identified in OsSh1 (Os03g0650000) is shown in bold. (B) Comparison of seed-shattering degree by breaking tensile strength (BTS) values in three introgression lines, $\mathrm{IL}(q S H 3-\mathrm{W}), \quad \mathrm{LL}(q S H 1-\mathrm{W})$, and $\mathrm{IL}(q S H 1-\mathrm{W}+q S H 3-\mathrm{W})$ in the Nipponbare genetic background. Data are mean \pm s.d. of four plants. n.s. and ${ }^{* *}$ indicate not significant and significant at the $1 \%$ level based on unpaired Student's t-test, respectively. $(C)$ Two types of constructs carrying qSH3 cDNA sequences of W630 and Nipponbare $\left(9 \mathrm{SH}_{3}{ }^{\mathrm{W} 630}\right.$ and $q S H 3^{N p b}$ ) driven by a $3-\mathrm{kb}$ region of the promoter used for transgenic analysis. $(D)$ The BTS values observed for the transformants with $q S H 3^{W 630}$ and $q S H 3^{N o b}$. Black triangles represent the average BTS value of IL( $q S H 1-W)$. 


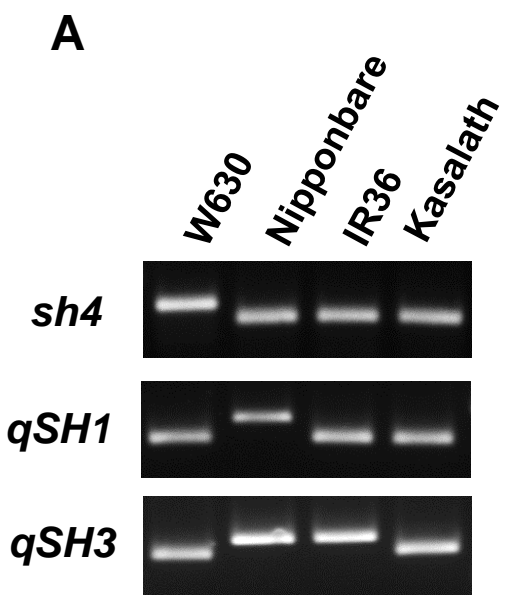

B
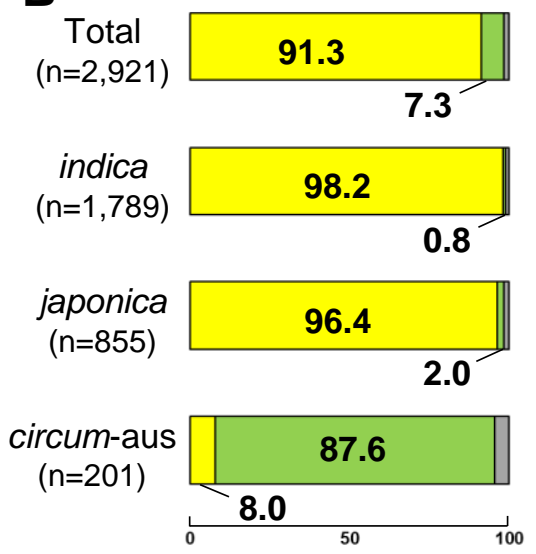

Allele frequency of $q S H 3$ causal SNP (\%)

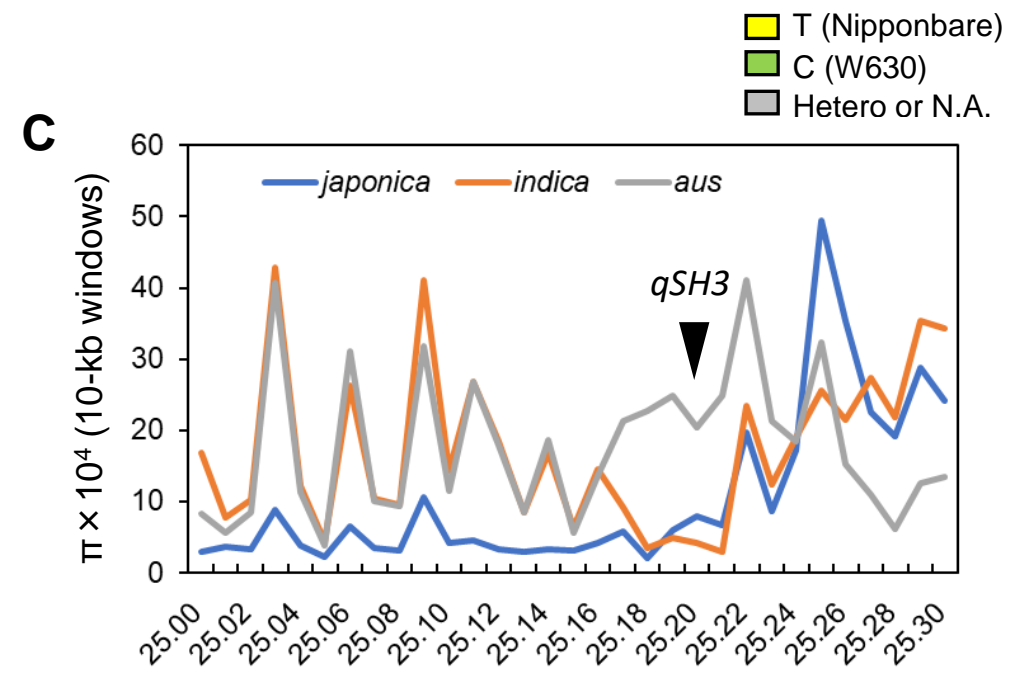

Position on chromosome $3(\mathrm{Mb})$

Fig. 2. Lineage-specific selection at $q S H 3$ locus in rice.

(A) Genotyping at $q S H 3$, sh4, and qSH1 for O. rufipogon W630, O. sativa japonica Nipponbare, indica IR36, and circum-aus 'Kasalath' based on the causal SNPs by dCAPS markers. (B) Allele frequency of $q S H 3$ causal SNP (\%) in cultivated rice based on the $3 \mathrm{~K}$ rice project data. Nipponbare type ('T') and W630 type ('C') are shown in yellow and green, respectively. (C) Nucleotide diversity ( $\pi$ ) observed for the domesticated rice in the physical position of 25.025.3 $\mathrm{Mb}$ on chromosome 3. A flanking region of the qSH3 locus (around $25.2 \mathrm{Mb}$ ) was substantially decreased in japonica and indica cultivars. $\pi$ was calculated in $10-\mathrm{kb}$ windows using SNPs data of the $3 \mathrm{~K}$ rice project (13). 
O. rufipogon W630
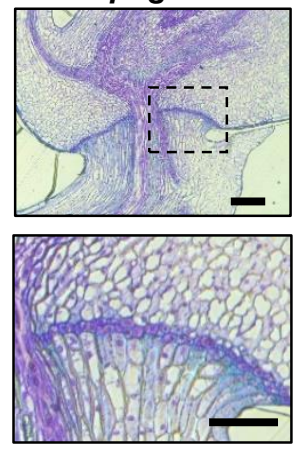

IL(sh4-N)
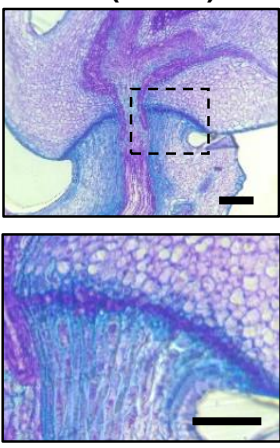

IL(qSH3-N)
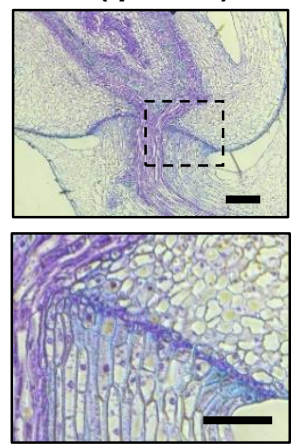
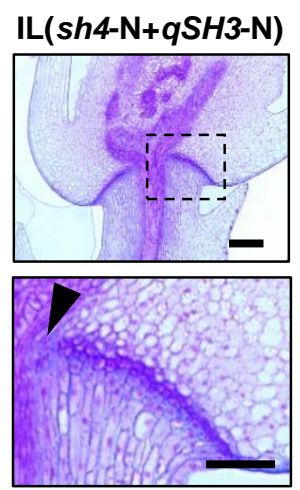

O. sativa Nipponbare
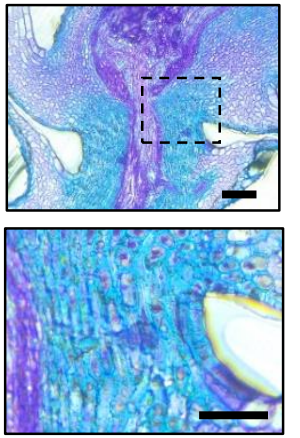

Fig. 3. Abscission layer formation partially inhibited by sh4 and $q S H 3$ in wild rice.

Abscission layer formation in O. rufipogon W630, IL(sh4-N), IL(qSH3-N), $\mathrm{IL}($ sh4-N+qSH3-N), and $\mathrm{O}$. sativa Nipponbare. Each enlarged section indicated by the dotted square is shown in the lower panel. A black triangle indicates an inhibited area of the abscission layer observed for IL(sh4$\mathrm{N}+q S H 3-\mathrm{N})$. Scale bars $=100 \mu \mathrm{m}$. 

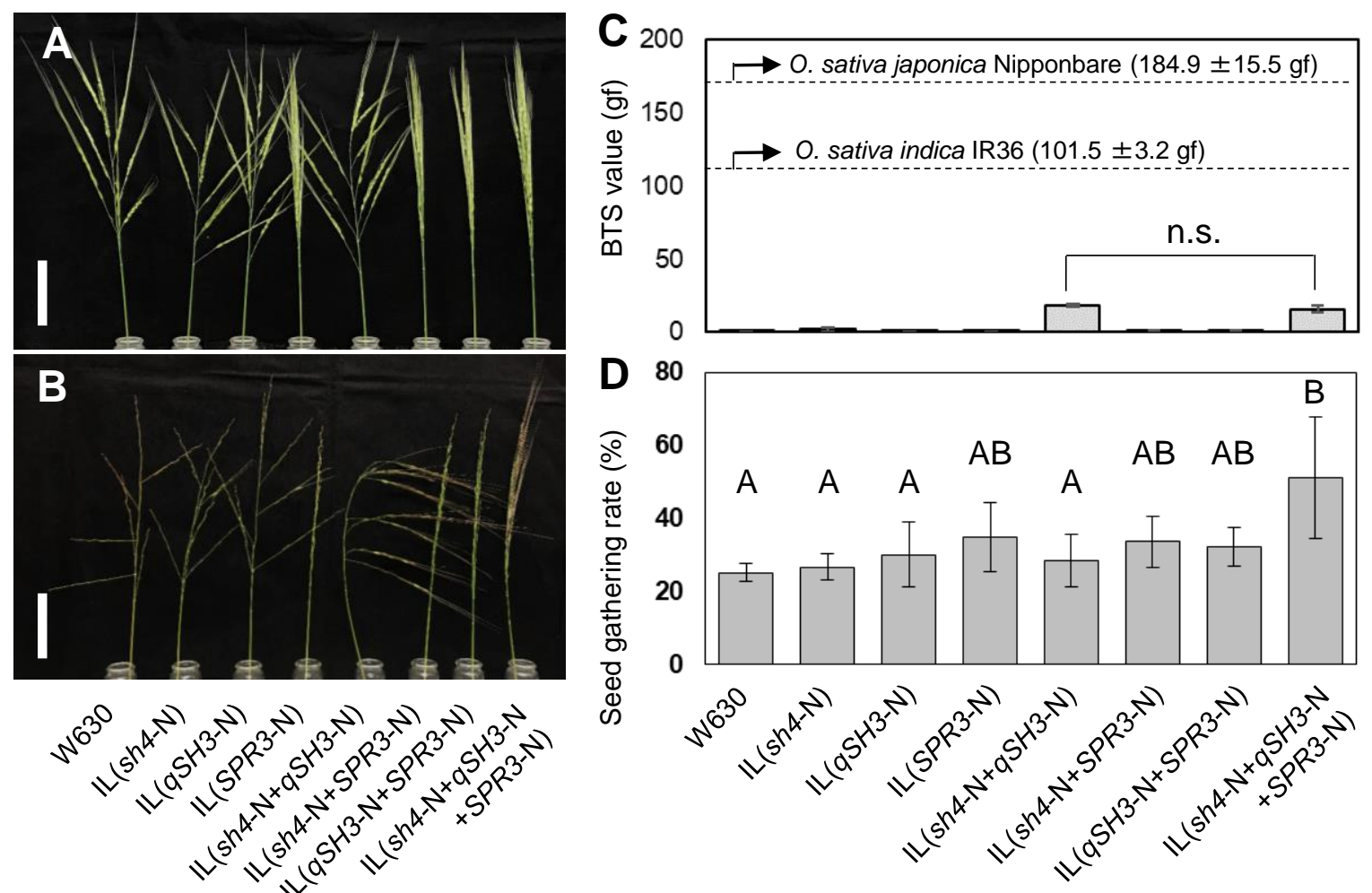

Fig. 4. Role of the sh4, $q S H 3$, and SPR3 mutations on the initial loss of seed shattering in rice domestication.

$(A, B)$ Panicle shape of the seven ILs at flowering $(A)$ and seed-maturation $(B)$ stages. Scale bars $=5 \mathrm{~cm}(C)$ Breaking tensile strength (BTS) values for the seven ILs. BTS values for $O$. sativa japonica Nipponbare and indica IR36 are shown as control. n.s. indicates not significant by unpaired Student's t-test. $(D)$ Seed-gathering rates (mean \pm s.d. of four plot replicates, with nine plants in each plot) for O. rufipogon W630 and the seven ILs. Mean values labelled with different letters are significantly different, whereas those with the same letters are not (Tukey's test with arcsine transformed values, $P<0.05)$. 


\section{Fig. 5}

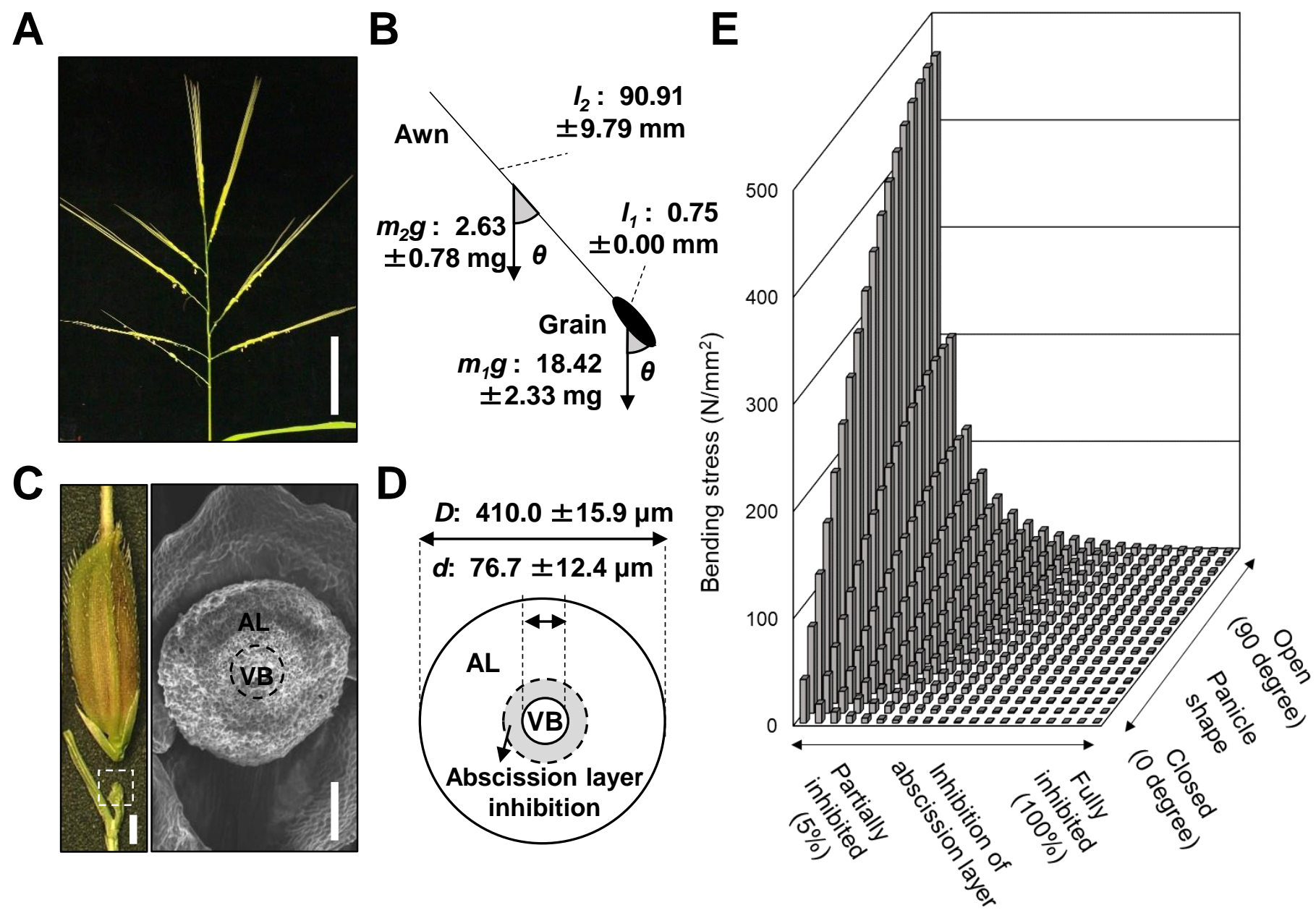

Fig. 5. Structural mechanics analysis of panicle shape and abscission layer inhibition for the initial loss of seed shattering.

$(A)$, Panicle shape of $O$. rufipogon W630. Bar $=5 \mathrm{~cm}$. (B) Schematic representation of awn and grain with panicle angle. $\theta$ represents the panicle angle. $l_{1}, l_{2}, m_{1} g$, and $m_{2} g$ indicate the length of grain, awn, weight of grain and awn, respectively. (C) Detachment of grain and pedicel (left) and scanning electron microscopy (SEM) analysis of the abscission layer of pedicel in $O$. rufipogon W630 (right). AL and VB indicate abscission layer and vascular bundle, respectively. Bars $=1 \mathrm{~mm}$ (left) and $100 \mu \mathrm{m}$ (right). (D) Schematic representation of the abscission layer. $D$ and $d$ indicate the diameter of abscission layer and vascular bundles, respectively. Dotted circle indicates the area of disrupted abscission layer. $(E)$ Simulation of the bending stress exerted on the spikelet base depending on panicle shape and abscission layer inhibition, with higher stress experience in open panicle with less inhibited abscission. 